\title{
A Hybrid Strategy for Reducing Feasible Convex Space and the Number of Variables for Solving a Conventional Large LP Model
}

\author{
Santosh Kumar \\ Department of Mathematics and Statistics \\ University of Melbourne, Parkville, Victoria, Australia \\ E-mail: santosh.kumarau@gmail.com \\ ${ }^{*}$ Correspondence author \\ Elias Munapo \\ School of Economics and Decision Sciences \\ North West University, Mafikeng Campus \\ Mafikeng, South Africa \\ E-mail: emunapo@gmail.com \\ 'Maseka Lesaoana \\ Department of Statistics and Operations Research \\ School of Mathematical and Computer Sciences \\ University of Limpopo, Turf loop Campus \\ Private Bag X1106, Sovenga 0727, South Africa \\ E-mail: Lesaoana.Maseka@ul.ac.za \\ Philimon Nyamugure \\ Department of Statistics and Operations Research \\ National University of Science and Technology \\ PO Box AC939, Ascot, Bulawayo, Zimbabwe \\ E-mail: philimon.nyamugure@nust.ac.zw \\ Nidhi Agarwal \\ Government Girls Senior Secondary School \\ Kota, Rajasthan, India \\ E-mail: nidhiagarawal20@gmail.com
}

(Received November 27, 2016; Accepted December 26, 2016)

\begin{abstract}
This paper considers a conventional linear programming model of ' $n$ ' variables and ' $m$ ' constraints. In the proposed method, we deal with $n_{1}$ number of variables, where $n_{1} \leq n$ and use a strategic move to reduce the feasible convex search space before embarking on the simplex method. The feasible space reduction process can be repeated, if desired.
\end{abstract}

Key words: Linear programming model, Simplex method, Feasible space reduction, Reduced number of variables.

\section{Introduction}

Many solution procedures have been developed for linear programming (LP) models. For example, see Dantzig (1963), Khachiyan (1980), Karmarkar (1984), and many variants of these approaches have been discussed in Forrest and Goldfrab (1992), Gay et al. (1998), Roos et al. (2006), Munapo and Kumar (2013), Small (1983), Vanderbei (2001, 2008), Zadeh (2009). Many other approaches have been suggested by Wright (1997, 1998), Ye (2011) and Zoutendijk (1960). Munapo and Kumar (2013) considered a LP model with non-negative coefficients, and developed 
International Journal of Mathematical, Engineering and Management Sciences

Vol. 2, No. 4, 213-230, 2017

https://dx.doi.org/10.33889/IJMEMS.2017.2.4-017

an iterative procedure to solve a large-scale LP model by transforming the given ' $n$ ' variable LP to a ' 2 ' variable LP. Computational experiments indicated that their approach performed better with regard to a large number of randomly generated large-scale LP problems. Later Munapo et al. (2014) reconsidered the same model and developed an iterative hybrid approach that uses the conventional simplex iterations to generate at least three consecutive extreme points improving the value of the objective function. These three extreme points were used to generate an interior point from which one moves in the direction of the normal to the given objective function hyperplane. The search is concluded when an optimal solution has been identified by the usual simplex optimality conditions.

In these two papers, Munapo and Kumar (2013) and Munapo et al. (2014), the large-scale LP has been assumed to have non-negative coefficients and a challenge has existed to extend these ideas to a general LP model without non-negative restrictions on coefficients. This paper aims to address this challenge.

The paper has been organized in 5 sections. Some essential ideas needed for developing the proposed approach have been discussed in Section 2, and the method has been presented in Section 3. Three numerical illustrations have been presented in Section 4, and finally the paper has been concluded in Section 5 .

\section{The LP Model and a Brief Review of Earlier Considerations}

\subsection{The LP Model}

In this paper, we consider a conventional LP model given as (1):

Maximize $\mathrm{Z}=C X$

Subject to: $A X \leq B$

where

$$
A=\left[\begin{array}{ccc}
a_{11} & \cdots & a_{1 n} \\
\vdots & \ddots & \vdots \\
a_{m 1} & \cdots & a_{m n}
\end{array}\right], \quad B=\left[\begin{array}{c}
b_{1} \\
\vdots \\
b_{m}
\end{array}\right], \quad C=\left[\begin{array}{lll}
c_{1} & \ldots & c_{n}
\end{array}\right], X=\left[\begin{array}{c}
x_{1} \\
\vdots \\
x_{n}
\end{array}\right]
$$

$x_{j} \geq 0$ for all $j$

\subsection{A Brief Review of Approaches Dealing with a LP Model with Non-Negative Coefficients}

The restricted LP model considered in Munapo and Kumar (2013) and Munapo et al. (2014) is given as (2):

Maximize $\mathrm{Z}=C X$

Subject to: $A X \leq B$

where

$$
\begin{aligned}
& A=\left[\begin{array}{ccc}
a_{11} & \cdots & a_{1 n} \\
\vdots & \ddots & \vdots \\
a_{m 1} & \cdots & a_{m n}
\end{array}\right], \quad B=\left[\begin{array}{c}
b_{1} \\
\vdots \\
b_{m}
\end{array}\right], \quad C=\left[\begin{array}{lll}
c_{1} & \ldots & c_{n}
\end{array}\right], X=\left[\begin{array}{c}
x_{1} \\
\vdots \\
x_{n}
\end{array}\right] \\
& a_{i j} \geq 0 \text { for all }(i, j), i=1,2, \ldots, m \text { and } j=1,2, \ldots, n ; \\
& b_{i} \geq 0 \text { for all } i, \quad c_{j} \geq 0 \text { and } x_{j} \geq 0 \text { for all } j
\end{aligned}
$$


International Journal of Mathematical, Engineering and Management Sciences

Vol. 2, No. 4, 213-230, 2017

https://dx.doi.org/10.33889/IJMEMS.2017.2.4-017

Note the difference between the LP models (1) and (2); in model (2), all coefficients are nonnegative. We briefly review strategies and limitations with regard to LP model (2) and develop new strategies to solve the LP model (1), which is free from non-negative restrictions on the coefficients.

Munapo and Kumar (2013) solved the LP model (2) by transforming the ' $n$-dimensional' problem to a '2-dimensional' problem, with a meaningful interpretation of the solution of the transformed 2-dimensional problem in the context of the given ' $n$-dimensional' problem. They defined two unknowns and found their optimal values by solving the 2-dimensional LP problem. These two values moved a starting search point $P_{i}$ on the surface of a constraint to an improved point $P_{i+1}$, which is also on the surface of another constraint. The purpose of moving from the point $P_{i}$ to $P_{i+1}$ was to improve the value of the objective function as much as possible. Thus, the value of the objective function at the point $P_{i+1}$ is greater than the value of the objective function at the point $P_{i}$. However, if optimality conditions are not satisfied at the point $P_{i+1}$, then this point $P_{i+1}$ takes the role of the point $P_{i}$ and the search for a new improved point $P_{i+1}$ starts again. This was an iterative process, details of which are given in Munapo and Kumar (2013). One of the directions among the two was moving in the direction of the normal of the objective hyper-plane. In this paper, we once again make use this direction.

Later, Munapo et al. (2014) developed a hybrid strategy to solve the LP model (2). They proposed that from an interior feasible point $I P_{0}$, one moves in the direction of the normal to the objective function as far as possible within the feasible region. Let this new position be denoted $I P_{1}$, which will either be an extreme point of the feasible region, or alternatively it may be a boundary point of the convex region of the LP structure defined by (2). Since the location of the new point is known, one can easily find the value of the objective function at that point. Let us denote the corresponding value of the objection function at this point by $Z_{I P_{1} \text {. }}$ If $I P_{1}$ is not an optimal solution, an additional condition:

$Z=\sum_{j=1}^{j=n} c_{j} x_{j} \geq Z_{I P_{1}}$

is added to the LP model (2), which reduces the feasible region. Once again, it is an iterative process. Details are given in Munapo et al. (2014).

In this paper, we propose to use this reduction in the feasible convex space and also eliminate requirement of non-negative conditions on various coefficients in a given LP model (Munapo and Kumar, 2013).

\subsection{Difficulties and Limitations}

(1) In the above two papers, it was assumed that all the coefficients of the given LP model were non-negative. However, in many real-life applications of the LP models, this condition may not be satisfied. Therefore, a need exists to develop a method for the general LP model.

(2) The transformation from ' $n$-variable' to a '2-variable' approach has a tendency to converge to the optimal solution. The convergence can be slow, but an approximate solution is very quick. Thus, the transformation approach has an advantage, not to be seen in any other LP approach, which can identify a quick approximate solution to the LP. 
International Journal of Mathematical, Engineering and Management Sciences

Vol. 2, No. 4, 213-230, 2017

https://dx.doi.org/10.33889/IJMEMS.2017.2.4-017

(3) The interior point in the hybrid approach Munapo et al. (2014) was generated by using three consecutive extreme points obtained by the simplex method. This interior point will be confined in the convex space generated by those three extreme points. Since the simplex search moves along the boundary of the convex feasible space generated by the LP constraints, the convex space formed by three extreme points will be such that two of its boundaries will be along the boundary of the feasible convex space. If we call these three consecutive extreme points as points 1,2 and 3, the plane joining points 1 and 3 will be through the feasible regions of the given LP model (2). Thus, the move in the direction of the normal to the objective function will increase the value in the objective function only if the normal direction remains in the feasible region of the LP model (2) and passes through the plane joining the points 1 and 3. Hence a better strategy is required.

This paper overcomes all these limitations and considers a general LP model without any nonnegative restriction on various coefficients. Details of the proposed approach are discussed in Section 3.

\section{Development of the Method for a Conventional LP Model (1)}

Let the number of variables and the number of constraints in the LP model (1) be denoted by ' $n$ ' and ' $m$ ' respectively. Without any loss of generality, let us consider $n_{1}$ of the $n$ variables $\left(n_{1}<\right.$ $n)$ to have positive coefficients in the objective function, and the remaining $\left(n-n_{1}\right)$ coefficients in the objective function to have negative values. We further assume, for ease of presentation, that the positive coefficients are associated with the first $n_{1}$ variables and that the negative coefficients are associated with the remaining variables $\left(n_{1}+1, n_{1}+2, \ldots, n\right)$. Furthermore, it is assumed that the variables $\left(n_{1}+1, n_{1}+2, \ldots, n_{2}\right)$ are such that one of the elements $a_{i j}<$ 0 for at least one $i, i=1,2, \ldots, m$ in the corresponding constraint columns $A_{j}$. The columns $A_{j}$ for the variables $\left(n_{2}+1, n_{2}+2, \ldots, n\right)$ are such that $a_{i j} \geq 0$ for all $i$. Thus, without any loss of generality, the LP model (1), can be expressed as given by (4).

Maximize $\mathrm{Z}=\sum_{j=1}^{n_{1}} c_{j} x_{j}-\sum_{n_{1}+1}^{n_{2}} c_{j} x_{j}-\sum_{n_{2}+1}^{n} c_{j} x_{j}$

Subject to: $\sum_{j=1}^{n_{1}} A_{j} x_{j}+\left(\sum_{j=n_{1}+1}^{n_{2}} A_{j} x_{j}\right)+\left(\sum_{n_{2}+1}^{n} A_{j} x_{j}\right) \leq B$

where

$$
\begin{aligned}
& A=\left[\begin{array}{ccc}
a_{11} & \cdots & a_{1 n} \\
\vdots & \ddots & \vdots \\
a_{m 1} & \cdots & a_{m n}
\end{array}\right]=\left[\begin{array}{llll}
A_{1} & A_{2} & \ldots & A_{n}
\end{array}\right], \quad B=\left[\begin{array}{c}
b_{1} \\
\vdots \\
b_{m}
\end{array}\right], \\
& C=\left[\begin{array}{lll}
c_{1} & \ldots & c_{n_{1}}, \ldots c_{n_{2+1}} \ldots c_{n}
\end{array}\right], \quad X=\left[\begin{array}{c}
x_{1} \\
\vdots \\
x_{n}
\end{array}\right] \\
& x_{j} \geq 0 \text { for all } j, c_{j} \geq 0 \text { for } j=1,2, \ldots, n_{1}, c_{j} \leq 0 \text { for } j=n_{1}+1, \ldots, n_{2}, n_{2}+1, \ldots, n . \\
& A_{j} \geq 0 \text { for } j=n_{2}, n_{2}+1, \ldots, n .
\end{aligned}
$$

From (4), a sub-problem is developed as given by (5).

Maximize $\mathrm{Z}=\sum_{j=1}^{n_{1}} c_{j} x_{j}$

Subject to: $A_{n_{1}} X_{n_{1}} \leq B, \sum_{j=1}^{n_{1}} c_{j} x_{j} \geq 0$ 
International Journal of Mathematical, Engineering and Management Sciences

Vol. 2, No. 4, 213-230, 2017

https://dx.doi.org/10.33889/IJMEMS.2017.2.4-017

where

$A_{n_{1}}=\left[\begin{array}{ccc}a_{11} & \cdots & a_{1 n_{1}} \\ \vdots & \ddots & \vdots \\ a_{m 1} & \cdots & a_{m n_{1}}\end{array}\right], \quad B=\left[\begin{array}{c}b_{1} \\ \vdots \\ b_{m}\end{array}\right], \quad C=\left[\begin{array}{lll}c_{1} & \cdots & c_{n_{1}}\end{array}\right], X_{n_{1}}=\left[\begin{array}{c}x_{1} \\ \vdots \\ x_{n_{1}}\end{array}\right]$

$x_{j} \geq 0$ for $j=1,2, \ldots, n_{1}$.

Note that in the sub-problem (5), all objective function coefficients are non-negative; hence the non-negative requirement with respect to objective coefficients is satisfied for the model (5). A move in the direction of the normal to the objective function of model (5) will be confined to the positive quadrant in the $n_{1}$-dimensional space. Also note that when we deal with model (5) instead of model (1), we are dealing with less number of variables.

Let us use the symbol $C R$ to denote the convex region defined by the linear constraints of the LP model (2). In the hybrid approach, one finds the interior point by averaging three successive extreme points obtained by the usual simplex iterations. These three extreme points will also form a convex region. Let $C R_{I}$ be the first convex region obtained from the first set of three extreme points. It may also be noted that subsequent extreme points will be based on other three extreme points, therefore, in a similar way, denote these subsequent convex regions by $C R_{i}, i=1$, $2, \ldots$ All these convex regions may not have any relationship among them, but they all share a common relationship with the $C R$, that is, each $C R_{i}$ is a sub-space of $C R$. The direction of the normal, which is a function of the given coefficients of the objective function, will increase the value of the objective function only if it can cross through the hyper-plane that shares the boundary with the feasible region on either side. This is possible by searching at least three extreme points, which are all adjacent to the current extreme point in different directions.

Thus, instead of finding three consecutive extreme points by the usual simplex iterations from the current location, we find ' $r$ ' number of extreme points from the same location, where $r \geq 3$. Note that these extreme points from the same location in ' $r$ ' random directions can be obtained in much less computational effort. Also the normal will be facing the boundary which is feasible on either side.

\subsection{A Few Observations with Regard to the Sub-Problem (5)}

Observation 1: Since the coefficients $c_{j}$ in problem (5) are non-negative, the normal direction to the hyper-plane representing the objective function of the sub-problem (5) will be confined to the positive quadrant in the $n_{1}$-dimensional space defined by (5). It is a similar situation that existed in earlier papers Munapo and Kumar (2013) and Munapo et al. (2014).

Observation 2: Consider that a feasible extreme point of the convex region defined by the given LP (5) is a known extreme point. If that point is not optimal, the non-optimality may be reflected by a large number of non-basic variables; each such variable reflecting non-optimality can generate a feasible extreme point of the convex region of (5). A random selection of ' $r$ ' variables can generate ' $r$ ' extreme points in different directions. An average of the selected extreme points will give rise to an interior point from where the direction of the normal will always be in the positive quadrant increasing the value of the objective function. Thus, instead of successive extreme points (like in the simplex approach), it is better to generate two or more extreme points, which are adjacent to the same feasible extreme point. Selection of these entering variables is random among those which qualify for an entry to the basis. Also note that the 
International Journal of Mathematical, Engineering and Management Sciences

Vol. 2, No. 4, 213-230, 2017

https://dx.doi.org/10.33889/IJMEMS.2017.2.4-017

computational effort required to generate the interior point in the above manner will require much less computational effort.

Observation 3: A known result in LP is that a LP is unbounded if minimum positive ratio does not exist and $c_{j}>0$. A similar but an inverse property of a LP could be that if a variable $x_{j}$ is such that $A_{j} \geq 0$ and $c_{j} \leq 0$, then the optimal value of the variable $x_{j}=0$. In other words the variable will consume resources without giving any positive return. Thus, such a variable will never qualify to enter the basis. Let us label this variable as a 'permanent non-basic' variable, and can then be removed from the LP model. Therefore, some variables in the model (1) may qualify to be a 'permanent non-basic' variable.

\section{Observation 4: Rounding the Interior Point to an Integer Point for Convenience}

Since in a LP model (5), the requirement of non-negative coefficients on the constraints set is not imposed, these coefficients may be positive or negative quantities, hence rounding up as well as rounding down will have to be checked for feasibility. Thus, rounding in this general model may not be desirable. Alternatively, the rounded values must be checked for feasibility with respect to all constraints.

\section{Observation 5: Maximizing the Value of the Objective Function}

Let the location of a known interior point be $I P_{k}$ and let the value of the objective function at this point also be known, which may be denoted by $Z_{I P_{k}}$. In order to achieve maximum increase in the value of the objective function, it is proposed to move from this location in the normal direction of the given objective hyper-plane. Thus, the new location is given by (6). Note that $C^{T} n_{i}$ is the normal direction of the given objective function of model (5).

$I P_{k}+\alpha C^{T_{n_{i}}}, \quad \alpha \geq 0$.

The scalar $\alpha \geq 0$ has to be calculated as the largest value such that $Z=C^{T n_{1}} X_{n_{1}}$ is maximized subject to:

$A_{\left(m X n_{i}\right)}\left(I P_{k}+\alpha C^{T_{n_{i}}}\right) \leq B, I P_{k}+\alpha C^{T_{n_{i}}} \geq 0$ or equivalently as shown in (7).

Maximize $\mathrm{Z}=C\left(I P_{k}+\alpha C^{T_{n_{i}}}\right)$

Subject to:

$\alpha A_{\left(m X n_{i}\right)} C^{T_{n_{i}}} \leq B-A_{\left(m X n_{i}\right)} I P_{k}, \alpha C^{T_{n_{i}}} \geq-I P_{k}$

The problem (7) is a one-variable LP, where the value of $\alpha$ is given by:

$\min _{i}\left(\frac{B-A_{\left(m X n_{i}\right)^{I P}}}{A C^{T n_{i}}}\right) \quad$ and $\quad \alpha C^{T_{n_{i}}} \geq-I P_{k}$

Once the value of $\alpha$ is known the new point with improved value of the objective function is a known point. The new improved feasible location may be an extreme point or may be a point on the boundary of a constraint of the given LP model (5). It is denoted by $I P_{k+1}$ and it can be determined from (9).

$I P_{k+1}=I P_{k}+\alpha C^{T_{n_{i}}}$ 
The new LP with reduced feasible space is given by (10). It is developed by adding the constraint (9) and the process can be repeated.

Maximize $\mathrm{Z}=C^{T_{n_{1}}} X_{n_{1}}=I P_{k+1}$

Subject to: $A_{m X n_{i}} X_{n_{1}} \leq B$

$Z \geq Z_{I P_{k+1}}, \quad X \geq 0$

Here $X_{n_{1}}=I P_{k+1}$.

Note that if $\alpha$ as obtained from (8) is equal to zero, it means that one has reached to the end of the feasible space in the normal direction of the objective function. If an optimal solution has not been identified, the LP model given by (10) involves less number of variables, and the feasible space is also reduced. The LP (10) can be solved using any package.

Observation 6: The new point $X=I P_{k+1}$ will always be the extreme point of the convex space defined by (10). In other words, the problem defined by (10) has a feasible region.

Proof: Note that the point defined by (9) will remain feasible to model (10) as all constraints of (5) are satisfied at this point and the additional constraint in the LP model (10) is also satisfied. Furthermore, it may be noted that at this point at least one of the constraints in the LP model (5) is active and the additional constraint in the model (10) is also active. Thus, it is an extreme point at the intersection of at least two active constraints of the model (10).

Observation 7: Once an optimal solution to the LP (5) is established, that solution can be used to establish the optimal solution to the model (2) by the application of column generation rule for the rest of the variables with negative coefficients in the objective function and also refer to information recycling discussed by Kumar $(2005,2006)$.

Observation 8: Given an ' $m$ ' constraint ' $n$ ' variable LP model to be solved, call it Problem 1. Let us construct another LP comprised of $n_{1}$ variables where $n_{1} \leq n$, call it Problem 2. If a variable $x_{j}$ belongs to both Problems 1 and 2 and has the same objective and constraint coefficients, then a basic feasible solution of the Problem 2 is also a basic feasible solution.

The proof is immediate as all variables not belonging to Problem 2 have an acceptable interpretation as non-basic variables with respect to the LP model (1). All constraints will remain satisfied.

Observation 9: For a given ' $m$ ' constraint ' $n$ ' variable LP model, the optimal solution of the LP comprised of ' $m$ ' constraints and $n_{1}$ variables where $n_{1} \leq n$ will form a lower bound on the optimal value of the given LP. The proof is obvious as any other additional given variable not among the $n_{1}$ variables can either increase the value of the objective function or remain non-basic at zero value.

\subsection{An Algorithm for Solving a LP Model (1) with Less Number of Variables and Reduced Feasible Space}

From the above discussion, one can generate an algorithm for solving a conventional large-scale LP by considering reduced feasible space and with less number of variables. The method is 
International Journal of Mathematical, Engineering and Management Sciences

Vol. 2, No. 4, 213-230, 2017

https://dx.doi.org/10.33889/IJMEMS.2017.2.4-017

comprised in two parts. Part 1 finds an optimal solution to the reduced LP model (5) and in Part 2, we find the optimality with respect to the given LP model (1).

\section{Part 1}

Step 1: Given a LP model (1). Check the objective function for positive coefficients. If all $c_{j}$ 's are non-negative, the number $n_{1}=n$ and $\left(n-n_{2}\right)=0$. Go to Step 2 .

If some of these coefficients are negative quantities, rearrange the given LP model in the structure of LP model (4), i.e. separating positive and negative coefficients of the objective function and rearrange the constraints accordingly to develop a sub-LP model of the form (4) and from that develop the LP model (5). Go to Step 2.

Step 2: Obtain an initial feasible point $\mathrm{IP}_{0}$ of the LP, which is given by $X=\left|\begin{array}{c}x_{1} \\ \vdots \\ x_{n_{1}}\end{array}\right|=\left|\begin{array}{c}0 \\ \vdots \\ 0\end{array}\right|$.

If the number of entering variables are more than a pre-assigned value ' $r \geq 3$ ', create at random ' $r$ ' feasible extreme points, which lead to an improved value of the objective function.

From these ' $r$ ' extreme points develop an interior point by averaging their values. Call this point $I P_{1}$.

Step 3: Move from the initial point $I P_{1}$ in the direction of the normal of the objective function within the feasible space as far as possible. Let this new feasible point be denoted by $I P_{2}$, and the value of the objective function at this point is denoted by $Z_{I P_{2}}$.

Step 4: Formulate a new LP problem (10):

Maximize $\mathrm{Z}=C X_{n_{1}}$

Subject to: $A X_{n_{1}} \leq B$

$C X_{n_{1}} \geq Z_{I P_{2}}, X_{n_{1}} \geq 0$, where $I P_{2}=I P_{1}+\alpha C^{T_{n_{1}}}$.

Step 5: Using any of the existing LP software, find an optimal solution to the LP in Step 4.

Step 6: Conclude the search process when the optimality conditions are satisfied, print the optimal solution to model (5).

\section{Part 2}

Consider the original LP model and select a variable $x_{j}$ for which $c_{j}<0$ for $j=n_{1}+1, \ldots, n$.

Step 7: Since $c_{j} \leq 0$, use column generation and find $Z_{j}-c_{j}=C_{B} B^{-1} A_{j}-c_{j}$. If $Z_{j}-c_{j}<$ 0 , carry out the usual simplex iteration and if $Z_{j}-c_{j} \geq 0$, go to Step 8 .

Step 8: Substitute $j=j+1$, if $j<n$, go to Step 7, else go to Step 9 .

Step 9: Print the optimal solution to the given LP model (1). 
International Journal of Mathematical, Engineering and Management Sciences

Vol. 2, No. 4, 213-230, 2017

https://dx.doi.org/10.33889/IJMEMS.2017.2.4-017

\section{Numerical Illustrations}

\section{Example 1: A Trivial Illustration}

$\operatorname{Max} z=x_{1}+x_{2}$

Subject to: $x_{1} \leq 1, x_{2} \leq 1,2 x_{1}-x_{2} \leq 1, x_{1}, x_{2} \geq 0$

This trivial problem has an optimal solution: $x_{1}=1, x_{2}=1, z=2$. See Fig. 1 for the solution. Since it is a two-variable problem, let us assign $r=2$.

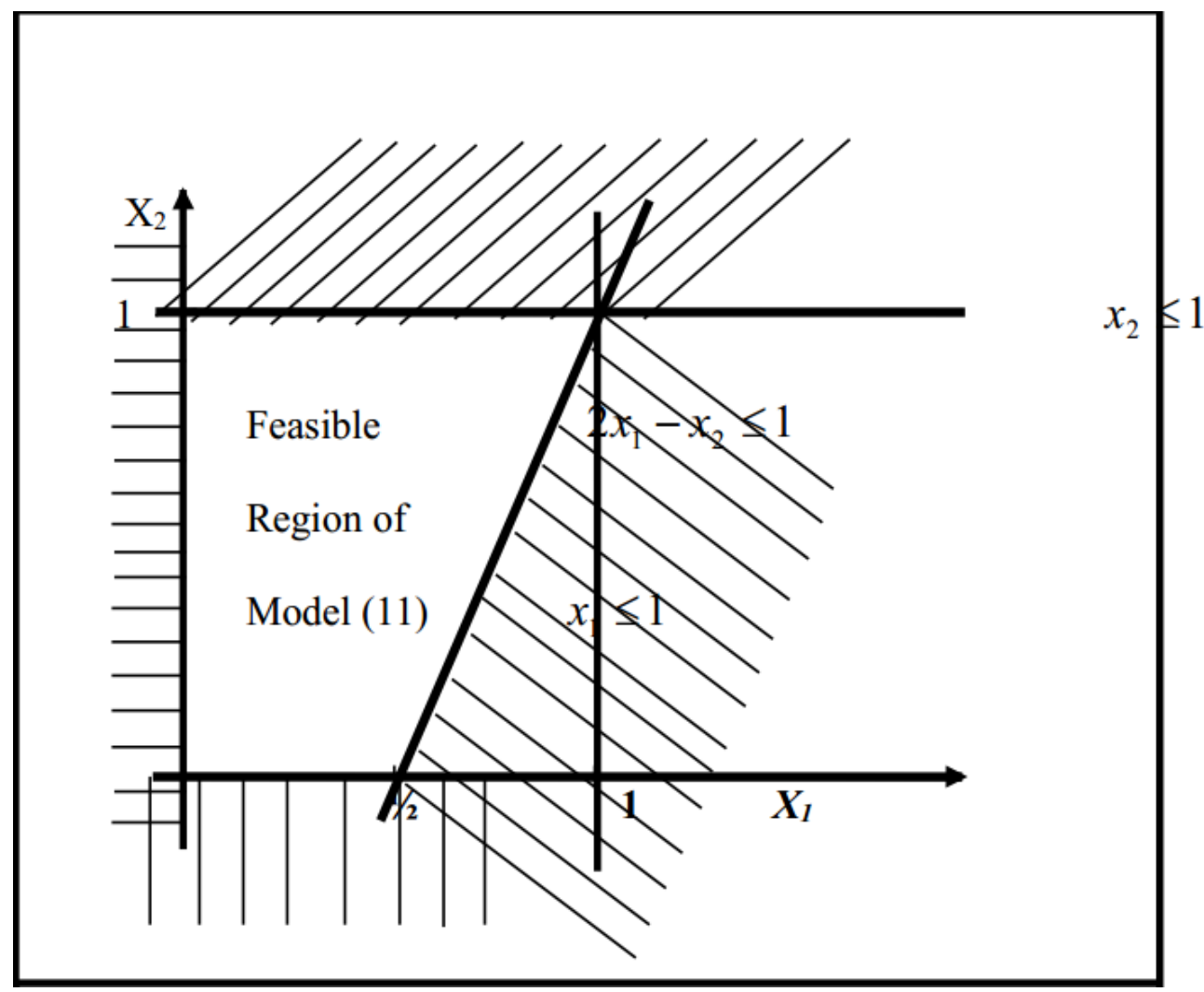

Fig. 1. Schematic presentation of the LP model (11)

An initial extreme point is $\mathrm{EP}_{0}$ which is the origin from where two possible extreme points that can be reached are: $\left(x_{1}, x_{2}\right)=(0.5,0)$ and $(0,1)$. The interior point $I P_{0}$ generated by these two points is given by $(0.25,0.5)$. The value of the objective function at this point is $Z_{I P_{0}}=0.75$. The improved point $I P_{1}$ can be obtained by solving the problem:

$\operatorname{Max} z=C\left(I P_{0}+\alpha C^{T}\right)=0.75+2 \alpha$

Subject to $\alpha\left[\begin{array}{cc}1 & 0 \\ 0 & 1 \\ 2 & -1\end{array}\right]\left[\begin{array}{l}1 \\ 1\end{array}\right] \leq\left[\begin{array}{l}1 \\ 1 \\ 1\end{array}\right]-\left[\begin{array}{cc}1 & 0 \\ 0 & 1 \\ 2 & -1\end{array}\right]\left[\begin{array}{c}0.25 \\ 0.5\end{array}\right]$

$$
\alpha\left[\begin{array}{l}
1 \\
1 \\
1
\end{array}\right] \leq\left[\begin{array}{c}
0.75 \\
0.5 \\
1
\end{array}\right]
$$

Hence $\alpha=0.5, I P_{1}=I P_{0}+\alpha C^{T}=\left[\begin{array}{c}0.75 \\ 1\end{array}\right]$ and $Z_{I P_{1}}=1.75$ 
International Journal of Mathematical, Engineering and Management Sciences

Vol. 2, No. 4, 213-230, 2017

https://dx.doi.org/10.33889/IJMEMS.2017.2.4-017

The modified LP with reduced space would be given by:

$\operatorname{Max} z=x_{1}+x_{2}$

Subject to: $x_{1} \leq 1, x_{2} \leq 1,2 x_{1}-x_{2} \leq 1, x_{1}+x_{2} \geq 1.75, x_{1}, x_{2} \geq 0$

For solution of (12), see Fig. 2.

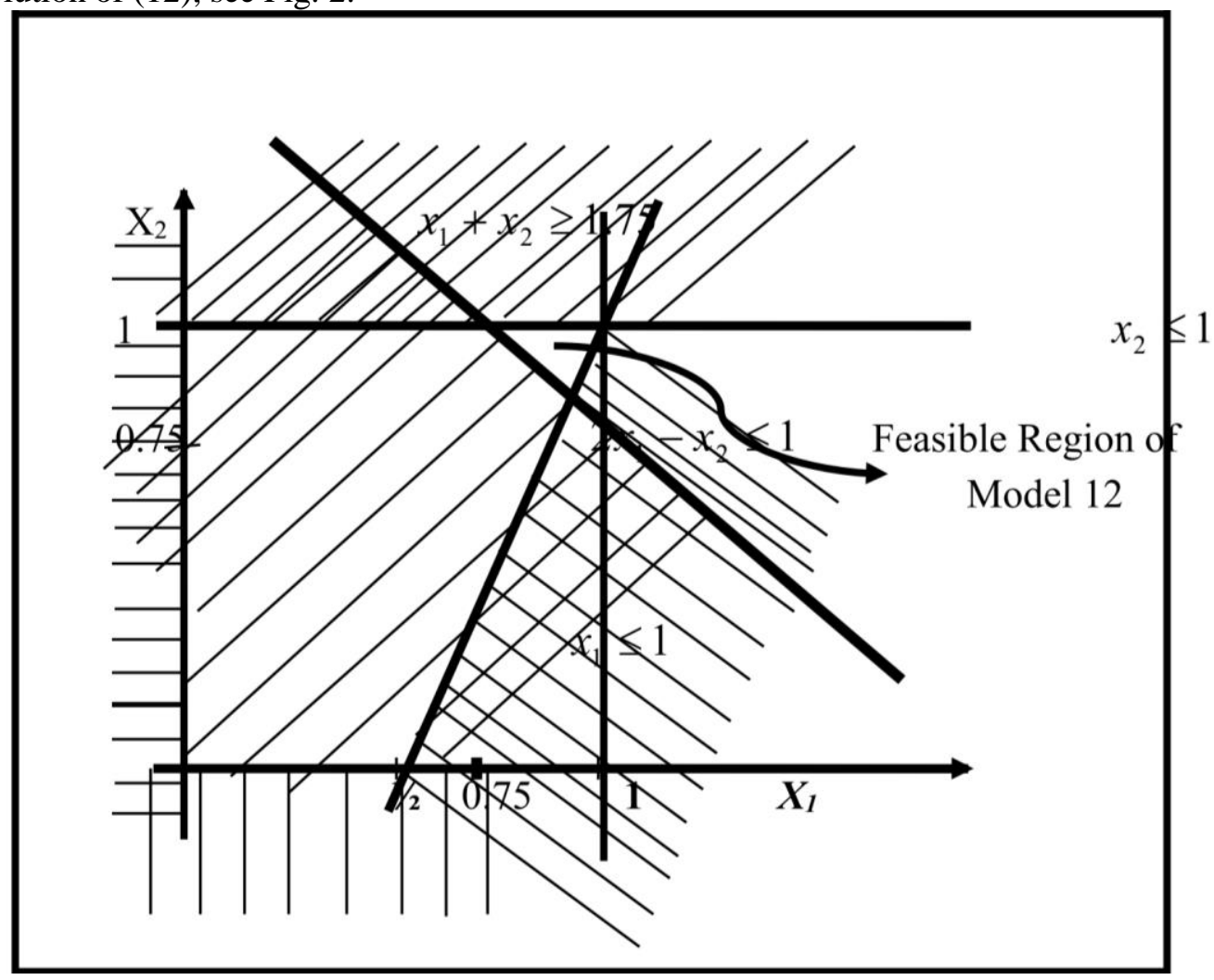

Fig. 2. Schematic presentation of the reduced feasible region after one move in the normal direction with respect to model (12)

The additional constraint reduces the feasible space, and the feasible solution to (12) is given in Table 1.

\begin{tabular}{|l|l|l|l|l|l|l|l|}
\hline & $\boldsymbol{x}_{\mathbf{1}}$ & $\boldsymbol{x}_{\mathbf{2}}$ & $\mathbf{S}_{\mathbf{3}}$ & $\mathbf{S}_{\mathbf{4}}$ & $\mathbf{S}_{\mathbf{5}}$ & $\mathbf{S}_{\mathbf{6}}$ & $\mathbf{R H S}$ \\
\hline $\mathbf{S}_{\mathbf{3}}$ & 0 & 0 & 1 & 0 & $-1 / 3$ & $1 / 3$ & $1 / 12$ \\
\hline $\mathbf{S}_{\mathbf{4}}$ & 0 & 0 & 0 & 1 & $1 / 3$ & $2 / 3$ & $1 / 6$ \\
\hline $\mathbf{X}_{\mathbf{1}}$ & 1 & 0 & 0 & 0 & $1 / 3$ & $-1 / 3$ & $11 / 12$ \\
\hline $\mathbf{X}_{\mathbf{2}}$ & 0 & 1 & 0 & 0 & $-1 / 3$ & $-2 / 3$ & $5 / 6$ \\
\hline $\mathbf{Z}_{\mathbf{j}} \mathbf{-} \mathbf{C}$ & 0 & 0 & 0 & 0 & 0 & -1 & 1.75 \\
\hline
\end{tabular}

Table 1. Feasible solution of the LP model (12)

For the optimal solution, variable $s_{6}$ enters the basis, pivot element will be $\left(s_{4}, s_{6}\right)$ in Table 1 , which results in an optimal solution. 
International Journal of Mathematical, Engineering and Management Sciences

Vol. 2, No. 4, 213-230, 2017

https://dx.doi.org/10.33889/IJMEMS.2017.2.4-017

\section{Example 2}

Consider the following Klee and Minty (1972) Cube

The Klee-Minty cube in ' $n$ ' dimension is given by:

$\operatorname{Max} z=\sum_{j=1}^{n} 10^{n-j} x_{j}$

Subject to: $2 \sum_{j=1}^{i-1} 10^{(i-j)} x_{j}+x_{i} \leq 100^{i-1}, x_{j} \geq 0, i=1,2, \ldots, n ; j=1,2, \ldots, n$.

For $n=3$, it will be:

$\operatorname{Max} z=100 x_{1}+10 x_{2}+x_{3}$

Subject to: $x_{1} \leq 1,20 x_{1}+x_{2} \leq 100,200 x_{1}+20 x_{2}+x_{3} \leq 10,000, x_{1}, x_{2}, x_{3} \geq 0$

In general, the simplex method can solve the Klee-Minty model in $\left(2^{n}-1\right)$ iterations and for $n=$ 3 , it will require 7 iterations. The optimal solution can be easily verified as: $x_{3}=10,000, x_{1}=x_{2}$ $=0$ and $z=10,000$. Now, we apply the proposed method discussed in this paper. The initial extreme point $\mathrm{EP}_{0}=\left(x_{1}, x_{2}, x_{3}\right)=(0,0,0)$. Let $r=3$.

Since $r=3$, the three possible extreme points from the initial point that can be reached are given by:

$(1,0,0),(0,100,0)$ and $(0,0,10000)$.

The interior point generated by these three points will be given by:

$I P_{0}=\left(\frac{1}{3}, \frac{100}{3}, \frac{10000}{3}\right)$

and the value of the objective function at this point will be given by:

$Z_{I P_{0}}=\frac{11100}{3}=3700$

To find the value of $\alpha$, we have

$$
A=\left|\begin{array}{ccc}
1 & 0 & 0 \\
20 & 1 & 0 \\
200 & 20 & 1
\end{array}\right|, B=\left|\begin{array}{c}
1 \\
100 \\
10,000
\end{array}\right|, C^{T}=\left|\begin{array}{c}
100 \\
10 \\
1
\end{array}\right|, I P_{1}=\left|\begin{array}{l}
x_{1} \\
x_{2} \\
x_{3}
\end{array}\right|=\left|\begin{array}{c}
\frac{1}{3} \\
\frac{100}{3} \\
\frac{10000}{3}
\end{array}\right|
$$

We therefore obtain

$$
\begin{aligned}
& A I P_{0}=\left|\begin{array}{c}
\frac{1}{3} \\
40 \\
7400
\end{array}\right|, A C^{T}=\left|\begin{array}{c}
100 \\
2010 \\
20201
\end{array}\right|, B-A I P_{0}=\left|\begin{array}{c}
0.67 \\
60 \\
2,600
\end{array}\right| . \\
& \text { Resulting in } \alpha=\min \left|\begin{array}{c}
100 \alpha \leq 0.67 \\
2010 \alpha \leq 60 \\
20201 \alpha \leq 2600
\end{array}\right|=\min (.0067, .0298, .1287)=.0067 .
\end{aligned}
$$


International Journal of Mathematical, Engineering and Management Sciences

Vol. 2, No. 4, 213-230, 2017

https://dx.doi.org/10.33889/IJMEMS.2017.2.4-017

Therefore, the improved interior point is

$I P_{2}=I P_{1}+\alpha C^{T}=\left|\begin{array}{c}1 / 3 \\ 100 / 3 \\ 10000 / 3\end{array}\right|+0.0067\left|\begin{array}{c}100 \\ 10 \\ 1\end{array}\right|=\left|\begin{array}{c}1.0 \\ 33.4 \\ 3333.34\end{array}\right|$.

The new constraint to be added will be

$100 x_{1}+10 x_{2}+x_{3} \geq Z_{I P_{1}}=3767.34$

Thus, the modified problem becomes:

$\operatorname{Max} z=100 x_{1}+10 x_{2}+x_{3}$

Subject to: $x_{1} \leq 1, \quad 20 x_{1}+x_{2} \leq 100, \quad 200 x_{1}+20 x_{2}+x_{3} \leq 10,000$,

$x_{1}, x_{2}, x_{3} \geq 0$

$$
100 x_{1}+10 x_{2}+x_{3} \geq 3767.34
$$

The feasible solution to (16) can be obtained by any method, and it is given in Table 2.

\begin{tabular}{|l|l|l|l|l|l|l|l|l|}
\hline $\mathbf{i} \mathbf{j}$ & $\mathbf{X}_{\mathbf{1}}$ & $\mathbf{X}_{\mathbf{2}}$ & $\mathbf{X}_{\mathbf{3}}$ & $\mathbf{S}_{\mathbf{1}}$ & $\mathbf{S}_{\mathbf{2}}$ & $\mathbf{S}_{\mathbf{3}}$ & $\mathbf{S}_{\mathbf{4}}$ & $\mathbf{R H S}$ \\
\hline $\mathbf{s}_{\mathbf{1}}$ & 1 & 0 & 0 & 1 & 0 & 0 & 0 & 1 \\
\hline $\mathbf{x}_{\mathbf{2}}$ & 20 & 1 & 0 & 0 & 1 & 0 & 0 & 100 \\
\hline $\mathbf{S}_{\mathbf{3}}$ & -100 & 0 & 0 & 0 & -10 & 1 & 1 & 5332.66 \\
\hline $\mathbf{X}_{\mathbf{3}}$ & -100 & 0 & 1 & 0 & -10 & 0 & -1 & 2767.34 \\
\hline $\mathbf{Z}-\mathbf{c}_{\mathbf{j}}$ & 0 & 0 & 0 & 0 & 0 & 0 & -1 & 3767.34 \\
\hline
\end{tabular}

Table 2. Feasible solution to problem (16)

For the optimal solution, one more pivot operation has to be performed on the element $\left(s_{3}, s_{4}\right)$ in Table 2, which will lead to the optimal solution.

\section{Example 3}

Consider a LP model as given by (17).

$\operatorname{Max} 2 x_{1}+5 x_{2}+3 x_{3}+4 x_{4}+2 x_{5}+3 x_{6}+2 x_{7}+x_{8}+2 x_{9}-x_{10}-2 x_{11}-3 x_{12}$

Subject to

$1 x_{1}+0 x_{2}-1 x_{3}+8 x_{4}-2 x_{5}+4 x_{6}+0 x_{7}+3 x_{8}+1 x_{9}+4 x_{10}-2 x_{11}+0 x_{12} \leq 24$

$2 x_{1}+2 x_{2}+0 x_{3}+3 x_{4}+3 x_{5}+0 x_{6}+0 x_{7}+4 x_{8}+0 x_{9}+0 x_{10}+1 x_{11}+1 x_{12} \leq 32$

$4 x_{1}+5 x_{2}+0 x_{3}+0 x_{4}+0 x_{5}+4 x_{6}+0 x_{7}+0 x_{8}+2 x_{9}+0 x_{10}+5 x_{11}+1 x_{12} \leq 30$

$3 x_{1}+0 x_{2}+3 x_{3}+2 x_{4}+3 x_{5}-2 x_{6}+4 x_{7}+2 x_{8}+0 x_{9}+1 x_{10}+1 x_{11}+0 x_{12} \leq 30$

$0 x_{1}+4 x_{2}+0 x_{3}-2 x_{4}+0 x_{5}+0 x_{6}+0 x_{7}-2 x_{8}+0 x_{9}+2 x_{10}+3 x_{11}+1 x_{12} \leq 22$

$2 x_{1}+1 x_{2}+4 x_{3}+1 x_{4}+2 x_{5}+0 x_{6}+5 x_{7}+1 x_{8}+0 x_{9}+0 x_{10}+2 x_{11}+3 x_{12} \leq 12$

$0 x_{1}+0 x_{2}+1 x_{3}+0 x_{4}+1 x_{5}+2 x_{6}+0 x_{7}+4 x_{8}+5 x_{9}+2 x_{10}+2 x_{11}+0 x_{12} \leq 28$

$1 x_{1}+0 x_{2}+0 x_{3}+3 x_{4}+0 x_{5}+1 x_{6}+1 x_{7}+2 x_{8}+6 x_{9}+1 x_{10}+1 x_{11}+2 x_{12} \leq 36$

$3 x_{1}+2 x_{2}+2 x_{3}+1 x_{4}-2 x_{5}+0 x_{6}-1 x_{7}+0 x_{8}+3 x_{9}+0 x_{10}+4 x_{11}+2 x_{12} \leq 18$

$x_{1}, x_{2}, \ldots, x_{12} \geq 0$ 
International Journal of Mathematical, Engineering and Management Sciences

Vol. 2, No. 4, 213-230, 2017

https://dx.doi.org/10.33889/IJMEMS.2017.2.4-017

Using a LP package, the optimal solution to the above LP is given by $Z=46.64$,

$x_{2}=4.72, x_{4}=3.12, x_{5}=2.08, x_{9}=3.2$

$x_{1}=x_{3}=x_{6}=x_{7}=x_{8}=x_{10}=x_{11}=x_{12}=0$.

Now we apply the method developed in this paper. Note that variables with positive coefficients in the objective function are given by:

$\left(x_{1}, x_{2}, x_{3}, x_{4}, x_{5}, x_{6}, x_{7}, x_{8}, x_{9}\right)$ and negative coefficients variables are: $\left(x_{10}, x_{11}, x_{12}\right)$.

The value of $n_{1}=9$. A sub-problem of 9-variables or less can be developed. Let us consider all these 9 variables to form a LP model similar to (5). This is given by (18).

$\operatorname{Max} 2 x_{1}+5 x_{2}+3 x_{3}+4 x_{4}+2 x_{5}+3 x_{6}+2 x_{7}+x_{8}+2 x_{9}$

Subject to

$$
\begin{aligned}
& 1 x_{1}+0 x_{2}-1 x_{3}+8 x_{4}-2 x_{5}+4 x_{6}+0 x_{7}+3 x_{8}+1 x_{9} \leq 24 \\
& 2 x_{1}+2 x_{2}+0 x_{3}+3 x_{4}+3 x_{5}+0 x_{6}+0 x_{7}+4 x_{8}+0 x_{9} \leq 32 \\
& 4 x_{1}+5 x_{2}+0 x_{3}+0 x_{4}+0 x_{5}+4 x_{6}+0 x_{7}+0 x_{8}+2 x_{9} \leq 30 \\
& 3 x_{1}+0 x_{2}+3 x_{3}+2 x_{4}+3 x_{5}-2 x_{6}+4 x_{7}+2 x_{8}+0 x_{9} \leq 30 \\
& 0 x_{1}+4 x_{2}+0 x_{3}-2 x_{4}+0 x_{5}+0 x_{6}+0 x_{7}-2 x_{8}+0 x_{9} \leq 22 \\
& 2 x_{1}+1 x_{2}+4 x_{3}+1 x_{4}+2 x_{5}+0 x_{6}+5 x_{7}+1 x_{8}+0 x_{9} \leq 12 \\
& 0 x_{1}+0 x_{2}+1 x_{3}+0 x_{4}+1 x_{5}+2 x_{6}+0 x_{7}+4 x_{8}+5 x_{9} \leq 28 \\
& 1 x_{1}+0 x_{2}+0 x_{3}+3 x_{4}+0 x_{5}+1 x_{6}+1 x_{7}+2 x_{8}+6 x_{9} \leq 36 \\
& 3 x_{1}+2 x_{2}+2 x_{3}+1 x_{4}-2 x_{5}+0 x_{6}-1 x_{7}+0 x_{8}+3 x_{9} \leq 18 \\
& \text { with variables } x_{j} \geq 0 \text { for } j=1,2, \ldots, 9 .
\end{aligned}
$$

For the LP model (18), we have:

$$
\begin{aligned}
& \mathrm{A}=\left|\begin{array}{ccccccccc}
1 & 0 & -1 & 8 & 5 & -2 & 4 & 3 & 1 \\
2 & 2 & 0 & 3 & 3 & 0 & 0 & 4 & 0 \\
4 & 5 & 0 & 0 & 0 & 4 & 0 & 0 & 2 \\
3 & 0 & 3 & 2 & 3 & -2 & 4 & 2 & 0 \\
0 & 4 & 0 & -2 & 0 & 0 & 0 & -2 & 0 \\
2 & 1 & 4 & 1 & 2 & 0 & 5 & 1 & 0 \\
0 & 0 & 1 & 0 & 1 & 2 & 0 & 4 & 5 \\
1 & 0 & 0 & 3 & 0 & 1 & 1 & 2 & 6 \\
3 & 2 & 2 & 1 & -2 & 0 & -1 & 0 & 3
\end{array}\right|, \quad B=\left(\begin{array}{l}
24 \\
32 \\
30 \\
30 \\
22 \\
12 \\
28 \\
36 \\
18
\end{array}\right), \\
& C=\left[\begin{array}{lllllllllll}
2 & 5 & 3 & 4 & 2 & 3 & 2 & 1 & 2
\end{array}\right], \\
& \text { and } \\
& X^{T}=\left[\begin{array}{lllllllll}
x_{1} & x_{2} & x_{3} & x_{4} & x_{5} & x_{6} & x_{7} & x_{8} & x_{9}
\end{array}\right] . \quad
\end{aligned}
$$


International Journal of Mathematical, Engineering and Management Sciences

Vol. 2, No. 4, 213-230, 2017

https://dx.doi.org/10.33889/IJMEMS.2017.2.4-017

The initial extreme point is

$$
E P_{0}=\left[\begin{array}{l}
x_{1} \\
x_{2} \\
x_{3} \\
x_{4} \\
x_{5} \\
x_{6} \\
x_{7} \\
x_{8} \\
x_{9}
\end{array}\right]=\left[\begin{array}{l}
0 \\
0 \\
0 \\
0 \\
0 \\
0 \\
0 \\
0 \\
0
\end{array}\right]
$$

Let $\mathrm{r}=3$. From this initial extreme point, we generate three extreme points with respect to variables $x_{2}, x_{3}$ and $x_{4}$. These points, expressed in variables $\left(x_{1}, x_{2}, \ldots, x_{9}\right)$ are given by:

$$
E P_{x_{2}}=\left[\begin{array}{c}
0 \\
5.2 \\
0 \\
0 \\
0 \\
0 \\
0 \\
0 \\
0
\end{array}\right], E P_{x_{3}}=\left[\begin{array}{l}
0 \\
0 \\
3 \\
0 \\
0 \\
0 \\
0 \\
0 \\
0
\end{array}\right] \text { and } E P_{x_{4}}=\left[\begin{array}{l}
0 \\
0 \\
0 \\
3 \\
0 \\
0 \\
0 \\
0 \\
0
\end{array}\right] \quad \text { resulting in } I P_{0}=\left[\begin{array}{c}
x_{1} \\
x_{2} \\
x_{3} \\
x_{4} \\
x_{5} \\
x_{6} \\
x_{7} \\
x_{8} \\
x_{9}
\end{array}\right]=\left[\begin{array}{c}
0 \\
1.8 \\
1 \\
1 \\
0 \\
0 \\
0 \\
0 \\
0
\end{array}\right] \text {. }
$$

The objective value at this point is $=Z_{I P_{0}}=1.8 .(5)+1 .(3)+1(4)=16$.

Since $C^{T}$ is the normal direction of the given objective function, we need to find the value of $\alpha$.

$$
\begin{aligned}
& \text { We find } A I P_{0}=\left[\begin{array}{ccccccccc}
1 & 0 & -1 & 8 & 5 & -2 & 4 & 3 & 1 \\
2 & 2 & 0 & 3 & 3 & 0 & 0 & 4 & 0 \\
4 & 5 & 0 & 0 & 0 & 4 & 0 & 0 & 2 \\
3 & 0 & 3 & 2 & 3 & -2 & 4 & 2 & 0 \\
0 & 4 & 0 & -2 & 0 & 0 & 0 & -2 & 0 \\
2 & 1 & 4 & 1 & 2 & 0 & 5 & 1 & 0 \\
0 & 0 & 1 & 0 & 1 & 2 & 0 & 4 & 5 \\
1 & 0 & 0 & 3 & 0 & 1 & 1 & 2 & 6 \\
3 & 2 & 2 & 1 & -2 & 0 & -1 & 0 & 3
\end{array}\right] \cdot\left[\begin{array}{c}
0 \\
1.8 \\
1 \\
1 \\
0 \\
0 \\
0 \\
0 \\
0
\end{array}\right]=\left[\begin{array}{c}
7 \\
6.6 \\
9 \\
5 \\
5.2 \\
6.8 \\
1 \\
3 \\
6.6
\end{array}\right] \\
& A C^{T}=\left[\begin{array}{ccccccccc}
1 & 0 & -1 & 8 & 5 & -2 & 4 & 3 & 1 \\
2 & 2 & 0 & 3 & 3 & 0 & 0 & 4 & 0 \\
4 & 5 & 0 & 0 & 0 & 4 & 0 & 0 & 2 \\
3 & 0 & 3 & 2 & 3 & -2 & 4 & 2 & 0 \\
0 & 4 & 0 & -2 & 0 & 0 & 0 & -2 & 0 \\
2 & 1 & 4 & 1 & 2 & 0 & 5 & 1 & 0 \\
0 & 0 & 1 & 0 & 1 & 2 & 0 & 4 & 5 \\
1 & 0 & 0 & 3 & 0 & 1 & 1 & 2 & 6 \\
3 & 2 & 2 & 1 & -2 & 0 & -1 & 0 & 3
\end{array}\right] \cdot\left[\begin{array}{l}
2 \\
5 \\
3 \\
4 \\
2 \\
3 \\
2 \\
1 \\
2
\end{array}\right]=\left[\begin{array}{l}
48 \\
36 \\
49 \\
33 \\
10 \\
40 \\
25 \\
33 \\
26
\end{array}\right]
\end{aligned}
$$


International Journal of Mathematical, Engineering and Management Sciences

Vol. 2, No. 4, 213-230, 2017

https://dx.doi.org/10.33889/IJMEMS.2017.2.4-017

$B-A P_{0}=\left[\begin{array}{l}24 \\ 32 \\ 30 \\ 30 \\ 22 \\ 12 \\ 28 \\ 36 \\ 18\end{array}\right]-\left[\begin{array}{c}7 \\ 6.6 \\ 9 \\ 5 \\ 5.2 \\ 6.8 \\ 1 \\ 3 \\ 6.8\end{array}\right]=\left[\begin{array}{c}17 \\ 25.4 \\ 21 \\ 25 \\ 16.8 \\ 5.2 \\ 27 \\ 33 \\ 11.2\end{array}\right]$

Since $\alpha=\min \left\{\left(\frac{B-I P_{k}}{A C^{T}}\right)\right\}=\left(\begin{array}{c}17 / 48 \\ 25.4 / 36 \\ 21 / 49 \\ 25 / 33 \\ 16.8 / 10 \\ 5.2 / 40 \\ 27 / 25 \\ 33 / 33 \\ 11.2 / 26\end{array}\right)=\left(\begin{array}{c}0.35 \\ 0.71 \\ 0.43 \\ 0.76 \\ 1.68 \\ 0.13 \\ 1.08 \\ 1.00 \\ 0.43\end{array}\right)=0.13$.

Using equation (8) and moving in the normal direction of the objective hyper-plane, the improved interior point is:

$I P_{1}=I P_{0}+\alpha C^{T}=\left[\begin{array}{c}0 \\ 1.8 \\ 1 \\ 1 \\ 0 \\ 0 \\ 0 \\ 0 \\ 0\end{array}\right]+0.13\left[\begin{array}{l}2 \\ 5 \\ 3 \\ 4 \\ 2 \\ 3 \\ 2 \\ 1 \\ 2\end{array}\right]=\left[\begin{array}{l}0.26 \\ 2.45 \\ 1.39 \\ 1.52 \\ 0.26 \\ 0.39 \\ 0.26 \\ 0.13 \\ 0.26\end{array}\right]$.

$Z_{I P_{1}}=2(0.26)+5(2.45)+3(1.39)+4(1.52)+2(0.26)+3(0.39)+2(0.26)+1(0.13)+2(0.26)$

$=26.88$.

Thus, a basic feasible solution to (18) is required in presence of the additional constraint given by (19).

$Z=2 x_{1}+5 x_{2}+3 x_{3}+4 x_{4}+2 x_{5}+3 x_{6}+2 x_{7}+x_{8}+2 x_{9} \geq 26.88\left(=Z_{I P_{1}}\right)$

The 9-variable problem (18) was solved using the LIPS LP package and the results were, as expected, the same as for the given 12-variable problem (17). These results were: 
International Journal of Mathematical, Engineering and Management Sciences

Vol. 2, No. 4, 213-230, 2017

https://dx.doi.org/10.33889/IJMEMS.2017.2.4-017

$x_{2}=4.72, x_{4}=3.12, x_{5}=2.08, x_{9}=3.2$ and $x_{1}=x_{3}=x_{6}=x_{7}=x_{8}=0, Z_{\text {opt }}=46.64$, although the result is optimal, but to establish the optimality of (17), we need inverse of the basic matrix of the 9 variable problem. From the LIPS LP package, the $\mathrm{B}^{-1}$ and set of basic variables were obtained as given in Table 3.

\begin{tabular}{|l|l|l|l|l|l|l|l|l|l|l|}
\hline Basic & $\mathbf{S}_{\mathbf{1 3}}$ & $\mathbf{S}_{\mathbf{1 4}}$ & $\mathbf{S}_{\mathbf{1 5}}$ & $\mathbf{S}_{\mathbf{1 6}}$ & $\mathbf{S}_{\mathbf{1 7}}$ & $\mathbf{S}_{\mathbf{1 8}}$ & $\mathbf{S}_{\mathbf{1 9}}$ & $\mathbf{S}_{\mathbf{2 0}}$ & $\mathbf{S}_{\mathbf{2 1}}$ & $\mathbf{R H S}$ \\
\hline $\mathbf{S}_{\mathbf{1 7}}$ & $2 / 75$ & 0 & $-4 / 3$ & 0 & 1 & $68 / 75$ & 0 & 0 & .89 & 9.36 \\
\hline $\mathbf{S}_{\mathbf{1 4}}$ & $-31 / 150$ & 1 & $-1 / 6$ & 0 & 0 & $-229 / 150$ & 0 & 0 & .18 & 6.96 \\
\hline $\mathbf{X}_{\mathbf{4}}$ & .12 & 0 & 0 & 0 & 0 & 0.08 & 0 & 0 & -.04 & 3.12 \\
\hline $\mathbf{S}_{\mathbf{1 6}}$ & .02 & 0 & 0.5 & 1 & 0 & -1.82 & 0 & 0 & -.34 & 1752 \\
\hline $\mathbf{X}_{\mathbf{2}}$ & $4 / 75$ & 0 & $1 / 3$ & 0 & 0 & $-14 / 75$ & 0 & 0 & -.24 & 4.72 \\
\hline $\mathbf{X}_{\mathbf{5}}$ & $-13 / 150$ & 0 & $-1 / 6$ & 0 & 0 & $83 / 150$ & 0 & 0 & .14 & 2.08 \\
\hline $\mathbf{S}_{\mathbf{1 9}}$ & $113 / 150$ & 0 & $11 / 6$ & 0 & 0 & $-433 / 150$ & 1 & 0 & -3.14 & 9.92 \\
\hline $\mathbf{S}_{\mathbf{2 0}}$ & .44 & 0 & 2 & 0 & 0 & -3.04 & 0 & 1 & -3.48 & 7.44 \\
\hline $\mathbf{X}_{\mathbf{9}}$ & $-2 / 15$ & 0 & $-1 / 3$ & 0 & 0 & $7 / 15$ & 0 & 0 & 0.6 & 3.2 \\
\hline $\mathbf{Z}_{\mathbf{j}}-\mathbf{c}_{\mathbf{j}}$ & $23 / 75$ & 0 & $2 / 3$ & 0 & 0 & $107 / 75$ & 0 & 0 & 0.12 & 46.64 \\
\hline
\end{tabular}

Table 3. Basic variables, solution and $\mathrm{B}^{-1}$ matrix

From Table 3, one can, for optimality, get values of

$z_{x_{10}}-c_{10}=\frac{167}{75}, z_{x_{11}}-c_{11}=\frac{604}{75}, z_{x_{12}}-c_{12}=\frac{614}{75}$ which confirms optimality of the given problem (17).

\section{Concluding Remarks}

(a) The above method is suitable for a conventional LP model of type (1). Advantages, if any, can be assessed only by developing and using an appropriate software to find computational efficiencies with respect to larger problems.

(b) From the initial point, a linear move in the normal direction is desirable if it increases the objective function value, the move can reduce number of simplex iterations.

(c) The proposed method is likely to converge to the solution faster than the simplex method by utilising movements in the normal direction to the objective function. A few iterations of moving in the normal direction give an approximate solution, which is not provided by any other method.

(d) Instead of dealing with the given problem of dimension $m X(n+m)$, in the proposed method we are dealing with $n_{1} X\left(n_{1}+m\right)$ problem, thus reducing pivoting computations in each iteration.

(e) Apply the ideas developed in this paper to solve a conventional integer and mix integer programming model discussed in Kumar and Munapo (2012), Nyamugure et al. (2017).

\section{Acknowledgements}

We are thankful to the referee for constructive suggestions. 
International Journal of Mathematical, Engineering and Management Sciences

Vol. 2, No. 4, 213-230, 2017

https://dx.doi.org/10.33889/IJMEMS.2017.2.4-017

\section{References}

Dantzig, G. B. (1963). Linear Programming and Extensions. Princeton, NJ.

Forrest, J. J., \& Goldfarb, D. (1992). Steepest-edge simplex algorithms for linear programming. Mathematical Programming, 57(1-3), 341-374.

Gay, D. M., Overton, M. L., \& Wright, M. H. (1998). A primal-dual interior method for nonconvex nonlinear programming. In Advances in Nonlinear Programming, 31-56. Springer US.

Karmarkar, N. (1984, December). A new polynomial-time algorithm for linear programming. In Proceedings of the Sixteenth Annual ACM Symposium on Theory of Computing, 302-311. ACM.

Khachiyan, L. G. (1980). A polynomial algorithm in linear programming. Soviet Mathematics Doklady, 20(1), 53-72.

Klee, V., \& Minty, G. J. (1972). How good is the Simplex algorithm? Inequalities, III, Proc. of the third symposium. Academic Press, 159-175.

Kumar, S. (2005). Information recycling mathematical methods for protean systems: A path-way approach. South African Journal of Industrial Engineering, 16(2), 81-101.

Kumar, S. (2006). Information recycling mathematical method: A path-way approach to geometric programs. South African Journal of Industrial Engineering, 17(2), 127-143.

Kumar, S., \& Munapo, E. (2012). Some lateral ideas and their applications for developing new solution procedures for a pure integer programming model, Keynote address, Proc. Of Herbal International Conference on Applications of Mathematics and Statistics for Intelligent solutions through Mathematics and Statistics, Edited by Marriappan, Srinivasan and Amritraj, Excell India Publisher, 1321.

Munapo, E., \& Kumar, S. (2013). Solving large-scale linear optimization problem with non-negative coefficients by transforming into a 2-variable LP problem. ASOR Bulletin, 32(1), 1-12.

Munapo, E., Kumar, S., Lesaoana, M., \& Nyamugure, P. (2014). Solving a large scale LP model with nonnegative coefficients: A hybrid search over the extreme points and the normal direction to the given objective function. ASOR Bulletin, 33(1), 11-23.

Nyamugure, P., Munapo, E., Lesaoana, 'M., \& Kumar, S. (2017). A heuristic for a mixed integer program using the characteristic equation approach. International Journal of Mathematical, Engineering and Management Sciences, 2(1), 1-16.

Roos, C., Terlaky, T., \& Vial, J. P. (2006). Interior point methods for linear optimization. Springer Science \& Business Media.

Small, S. (1983). On the average speed of the simplex method in linear programming. Mathematical Programming, 27, 241-262.

Vanderbei, R. J. (2001). Linear programming: foundations and extensions. International Series in Operations Research \& Management Science.

Vanderbei, R. J. (2008). Linear programming: foundations and extensions. Third Edition, Springer, pp 48.

Wright, M. H. (1998). The interior-point revolution in constrained optimization. In High Performance Algorithms and Software in Nonlinear Optimization, 24, 359-381. Springer US.

Wright, S. (1997). Primal-dual Interior methods. SIAM, Philadelphia.

Ye, Y. (2011). Interior point algorithms: theory and analysis, 44. John Wiley \& Sons. 
International Journal of Mathematical, Engineering and Management Sciences

Vol. 2, No. 4, 213-230, 2017

https://dx.doi.org/10.33889/IJMEMS.2017.2.4-017

Zadeh, N. (2009). What is the worst case behavior of the simplex algorithm? Polyhedral computation, 48, 131-143.

Zoutendijk, G. (1960). Methods of feasible directions: a study in linear and non-linear programming. Elsevier. 